\section{Weiterbildung in Hausarztpraxen}

Eigentlich ist es allen klar: Das Spenglerhandwerk lernt man beim Spengler, das Backen beim Bäcker, das Mauern beim Maurer und das Metier des Hausarztes in der Hausarztpraxis. Auch das KVG hat dies erkannt und schreibt vor, dass ein Teil der Weiterbildung der Ärzte im Bereich der ambulanten Patientenbetreuung zu erfolgen hat.

Alles klar?

Ein Projekt zur Weiterbildung in Hausarztpraxen (Praxisassistenz) läuft unter Federführung des Kollegiums für Hausarztmedizin seit gut drei Jahren mit grossem Erfolg, je einen Bericht über den Verlauf und die Evaluation finden Sie in dieser Ausgabe [1, 2]: alle sind begeistert.

Alles klar?

In den EU-Richtlinien über die Weiterbildung in Allgemeinmedizin ist die Praxisassistenz verankert, in Kanada, Australien und den USA ist sie etabliert. Alles klar?

Alles klar? Eben nicht!!!!

Die Logistik ist im Aufbau, fast wöchentlich werden neue Lehrpraxen anerkannt, die Administration wird laufend optimiert, dieses Problem wird gelöst.

Das Projekt kann dank eines teilweisen Lohnverzichtes der Assistenten von FMH, VSAO, den Grundversorgergesellschaften und den Praxisinhabern getragen werden. Dieses finanzielle Engagement wird auch in Zukunft unabdingbar notwendig sein.

Leider ungelöst ist jedoch das Problem der Finanzierung einer Praxisassistenz für ALLE zukünftigen Grundversorger (oder noch besser alle praktizierenden Ärzte).
Der gesetzlichen Regelung, dass die Weiterbildung vom Weiterzubildenden zu bezahlen sei, wird im Spital durch den Mix aus Weiterbildung und Dienstleistung Folge geleistet. Wie die Evaluation des Pilotprojektes zeigt, ist dies jedoch in der Praxis nicht möglich, da die Anwesenheit eines Praxisassistenten in der Regel nicht zu einer Vermehrung der Patientenkontakte und eher zu einer zeitlichen Mehrbelastung des Praxisinhabers führt.

Da die Studie eindrücklich beweist, dass sich die zukünftigen Grundversorger nach absolvierter Praxisassistenz eindeutig besser auf ihre zukünftige Tätigkeit vorbereitet fühlen, müssten doch eigentlich alle Parteien des Gesundheitswesens grosses Interesse daran haben, dass möglichst in Zukunft alle praktizierenden Ärztinnen und Ärzte von dieser Berufsvorbereitung profitieren können, die das Gesetz - wie schon bemerkt - eigentlich vorschreibt.

Oder darf auch hier Qualität nichts kosten?

Ich möchte jedenfalls allen Politikerinnen und Politikern, Sanitätsdirektorinnen/-direktoren, Krankenkassenverantwortlichen, Finanzchefs der Pharmafirmen und allen andern Skeptikern die Lektüre der erwähnten Berichte sehr empfehlen. Eine Investition in die Optimierung der Qualität unserer medizinischen Grundversorgung lohnt sich mit Sicherheit!

Dr. med. Marc Müller, Präsident Kollegium für Hausarztmedizin

1 Rindlisbacher R. Weiterbildung in Hausarztpraxen. Aufbau und Durchführung des dreijährigen Pilotprojektes 1. Juli 1998 - 30. Juni 2001. Schweiz Ärztezeitung 2002;83(9):407-9.

2 Schläppi P, Hofer D, Bloch R. Lernform Praxisassistenz bewährt sich. Evaluation des dreijährigen Pilotprojektes "Weiterbildung in Hausarztpraxen (Praxisassistenz)» des Kollegiums für Hausarztmedizin KHM in Zusammenarbeit mit FMH, VSAO und SAMW. Schweiz Ärztezeitung 2002;83(9):410-6. 